\title{
Atherogenesis, the oxidative LDL modification hypothesis revisited
}

\author{
Dov Lichtenberg*, Ilya Pinchuk \\ Department of Physiology and Pharmacology, Sackler School of Medicine, Tel-Aviv University, Tel-Aviv, Israel \\ Email: ${ }^{*}$ physidov@post.tau.ac.il
}

Received 23 September 2013; revised 25 November 2013; accepted 15 December 2013

Copyright (C) 2013 Dov Lichtenberg, Ilya Pinchuk. This is an open access article distributed under the Creative Commons Attribution License, which permits unrestricted use, distribution, and reproduction in any medium, provided the original work is properly cited.

\section{ABSTRACT}

The commonly-accepted "oxidized LDL hypothesis of atherogenesis" is based on a large number of indirect evidence that shows that oxidatively-modified LDL plays a role in atherogenesis. Yet, the exact role is not clear. Some researchers think that oxidatively modified biomolecules initiate atherogenesis; others believe that they "only" promote this multifactorial process. Regardless of the exact mechanism responsible for the effect of peroxidation on atherogenesis, the "oxidative theory of AS" is apparently inconsistent with the results of meta-analysis, in which (the "expected") significant correlation between CVD and oxidative stress (OS) was found only when the $O S$ was evaluated on the basis of the plasma concentrations of malondialdehyde (MDA), often based on the concentration of thiobarbituric acid reactive substances (TBARS). Notably, even this association is questionable due to 1) poor reliability of the laboratory assay of MDA and 2) possible publication bias. Hence, it appears that the commonly accepted paradigm regarding the role of oxidative damage in the pathogenesis of CVD has been overestimated. Furthermore, the hypothesis is apparently inconsistent with the disappointing results of most of the clinical trials that were designed to reduce $O S$ by means of supplementation of antioxidants, mostly vitamin E. These apparent inconsistencies do not contradict the oxidative modification hypothesis of AS. The source of the apparent contradictions is probably the oversimplified considerations on which the predictions have been based. Many reasonable arguments can be raised to explain the apparent contradictions, which means that our current knowledge is insufficient to test the relationship of oxidative stress to cardiovascular disease.

Keywords: ROS; Free Radicals; Atherosclerosis;
Oxidative Stress; Antioxidants

\section{INTRODUCTION}

Reactive oxygen species (ROS) and reactive nitrogen species (RNS) play essential and diverse roles in humane physiology [1-8]. They contribute to processes such as apoptosis and mitogenesis, they help maintaining cell number homeostasis and they play a central role in immune responses and cell signaling, particularly at the level of redox modulation. By contrast, excessive production of these reactive species (RONS), particularly free radicals, damages DNA, proteins and lipids, thus being cytotoxic [9-11]. The concentration of RONS is therefore a critical parameter in determining their ultimate cellular response. ROS-induced stimulation of cells has been shown to result in changes of the cellular redox potential due to interruption of redox balance, which may adversely affect cell function. Consequently, the dependence of the response on the concentration of RONS is unpredictable. In fact, the prevailing reputation of free radicals is that they are the "bad guys" responsible for aging [12-16] and for many diseases [17-19].

The oxidative modification hypothesis of atherogenesis, first proposed by Steinberg et al. in 1989, is that atherogenesis is a consequence of oxidative modification of LDL [20]. The physiological role of LDL is to carry cholesterol from the liver to cells throughout the body. Internalization of oxidized LDL into cells, including endothelial cells and macrophages, differs from internalization of native LDL. Whereas native LDL is recognized and internalized into cells by way of the LDL receptor, oxidized LDL is recognized by the so-called scavenger receptors, which, unlike the LDL receptor, are not downregulated $[21,22]$. The liver is very rich in scavenger receptors, so that soon after intravenous injection of oxidized LDL it disappears from the blood, becoming accumulated in endothelial cells and macrophages. As a result, these cells become "foam cells" and die, thus 
forming atherotic plaques [23-25].

The peroxidation-induced atherogenesis hypothesis [20] inspired the National Heart, Lung and Blood Institute to fund a workshop of a group of 30 distinguished specialists in various aspects of the relevant fields to review all of the evidence available up to that time, including some epidemiologic data [26]. The panel found that all the available knowledge is compatible with the oxidative modification hypothesis. Hence, the panel's final recommendation was that studies utilizing naturally-occurring antioxidant vitamins (e.g. vitamin E, Bcarotene and vitamin C) should proceed [27,28]. In view of this conclusion, the oxidation hypothesis of atherosclerosis became the most commonly accepted paradigm.

Since the removal of oxidized LDL by the scavenger receptors is rapid, minimally oxidized LDL disappears from the circulation slower than more oxidized LDL. Consequently, the level of oxidized LDL in the blood is low and much of it is minimally oxidized LDL [29]. Notably, the autoantibody against oxidized LDL investigated by Witztum et al. demonstrated that even minor modifications in the structure of LDL make it immunogenic and that this specific antibody can be observed in arterial lesions, in agreement with the notion that most of the AS-relevant oxidation probably occurs in the artery wall itself [29]. Notably, in the presence of copper, the LDL-associated antioxidants become oxidized and only then, rapid oxidation of LDL lipids (both fatty acids and cholesterol) begins [30].

The obvious implication of this mechanism was that if peroxidation of LDL is the cause of atherogenesis, antioxidants should help prevent it. Unfortunately, the results of clinical trials were disappointing. Specifically, although several trials indicated that vitamin E supplementation reduces the rate of both nonfatal MI and Nonfatal Stroke, in other trials vitamin E had no significant effect on different cardiac end points. The important argument of the supporters of vitamin E supplementation is that some people benefit from it, whereas "if it does not help, it does not hurt" [31]. In the words of William A. Pryor: "In view of the very low risk of reasonable supplementation with vitamin E, some supplementation appears prudent now" [31].

Being aware of the conflicting evidence, Witztum and Steinberg recall that cardiovascular disease (CVD) is a complex and multifactorial inflammatory disease associated with gradual progression of plaques. Furthermore, they raised the possibility that the "Oxidative modification Hypothesis" may not hold for humans and concluded that we need to design clinical trails sensitive to early lesions [29]. Moreover, in 2004, Stocker and Keaney, in their review on the "Role of oxidative modifications in Atherogenesis", specifically stated that it is not clear whether oxidative events are a cause or a result of
AS [32]. At about the same time, two independent metaanalyses concluded that vitamin E supplementation results in higher mortality [33,34], which raised the question whether antioxidant supplementation is "Good in Theory, but is the theory good?" [35].

The possibility that the theory is not good can not be ruled out, but the apparent contradiction between the predicted and observed effects of vitamin E can be explained differently. Specifically, the lack of beneficial effect(s) of vitamin E supplementation may be due to at least one of many processes discussed in this critical, non-comprehensive review. For this discussion to be understood, we must first briefly describe the physiological roles of RONS and the evidence for their involvement in atherogenesis. Next, we relate to the available data on the oxidative status in CVD patients in comparison to matched controls. We then take a closer look at the intervention studies, particularly vitamin E supplementation trials. In an attempt to gain understanding of the apparent inconsistencies between the hypothesis and the experimental results, we relate to a variety of possibilities with emphasis on the very complex (both protective and atherogenic) role of HDL and of lipoprotein-associated enzymes on the peroxidation of LDL lipids. Based on this critical review of available data, we can only conclude that the specific roles of LDL oxidative modification in atherosclerosis are in a state of uncertainty.

\section{THE PHYSIOLOGICAL ROLE OF REACTIVE OXYGEN AND NITROGEN SPECIES}

For many years, OS has been commonly regarded a measure of a person's probability to suffer from oxidative damages. Thus, OS have been commonly blamed for being involved in the pathogenesis of many diseases and antioxidants predicted to be good to us $[9,18]$. Now we realize that "Reactive oxygen species are 'double-edged swords' in cellular processes: low-dose cell signaling versus high-dose toxicity" $[10,11]$. Hence, "the dose response curve is unpredictable" [10]. In other words, the effects of "Free radicals and antioxidants in (both) normal physiological functions and human disease" [8] depend upon their concentrations. At moderate concentrations, both the production and metabolism of RONS are tightly controlled [6] and cellular 'redox homeostasis' is retained.

Under homeostatic conditions, ROS act as secondary messengers in intracellular signaling cascades, including the ROS-induced cell signaling and particularly the signaling for skeletal muscle adaptation [3]. By that, ROS contribute to the defense against infectious agents, as well as to other immune reactions, apoptosis and mitogenesis and anti-tumourigenic species. The claims made 
by Ristow's group [16] that "there is experimental basis to question Harman's Free Radical Theory of Aging" [12] and that "the existing data suggest that ROS act as essential signaling molecules to promote metabolic health and longevity" [16], have yet to be evaluated but can not be ruled out.

By contrast, under certain conditions free radicals may induce and maintain the oncogenic phenotype of cancer cells and this is merely one of many deleterious effects of over produced ROS, including aging and human diseases, particularly cancer, cardiovascular disease, atherosclerosis, hypertension, ischemia/reperfusion injury, diabetes mellitus, Alzheimer's disease and Parkinson's disease $[5,18,19]$. Admittedly, it is not clear whether excessive formation of free radicals is the primary cause of any of these diseases or merely a result of inflammation or/and tissue injury. Yet, it is commonly believed that the ROSinduced damage to cell components, including membrane lipids, proteins and DNA contributes to all these pathologies.

In short, the effects of RONS on both human function and diseases depend in a complex, non-monotonic fashion on their characteristics and concentrations in the relevant compartment. These, in turn depend, in a complex, interrelated fashion on a large number of factors. Hence, at the present time we do not have a way to predict whether a given change of the redox status is likely to promote or retard our health.

\section{EVIDENCE FOR THE INVOLVEMENT OF FREE RADICALS IN ATHEROGENESIS}

The "oxidative modification-hypothesis of AS" was originally based on five findings in Steinberg's group $[20,29]$ :

1) That "incubation of endothelial cells in culture with high concentrations of LDL led to cell death, whereas the LDL re-isolated from the medium of a cell culture after incubation was markedly altered, such that it was taken up by monocytes/macrophages in culture much faster than native (normal) LDL [36].

2) That the major change that occurred during the incubation did not take place when the medium in which the cells were grown did not contain transition metal ions [37].

3) That addition of antioxidants to cells in plasmacontaining cultures prevented the changes in the LDL. Specifically, addition of vitamin E could completely prevent oxidation of LDL induced by incubation with the cells [37].

4) That oxidized LDL is a chemoattractant for blood monocytes, which helps recruiting them into a developing lesion [24].

5) That oxidized LDL inhibits the motility of tissue macrophages, which would tend to trap such cells in the artery wall once they got there [25].

At that time, there were several preliminary evidences that treatment of rabbits with several antioxidants reduced the rate of atherogenesis [38].

After the publication of the oxidative modification hypothesis of AS, a large number of supporting, mainly indirect lines of evidence appeared, including evidence based on the composition of atherotic plaques [39] and data on the effect of antioxidants on the rate of atherogenesis in primates [28].

Today, we have many more lines of evidence for the involvement of LDL oxidation in atherogenesis. First, we know that there are at least ten different ways by which different oxidation products of LDL can be atherogenic via various mechanisms $[4,29]$ and that the mode of LDL oxidation affects the biological effects of the oxidation products, as described by Levitan et al. in their comprehensive review [40]. Although these authors found "a significant degree of specificity to different forms of oxLDL", they note in their concluding remarks that we do not know "which of the oxLDL forms has the most pronounced effect on the development of the AS lesions" and that in spite of "the considerable progress made in recent years...there are still unresolved issues", which are difficult to resolve because the studied oxLDL preparations are "poorly defined" [40].

We also know that other processes beside oxidation can also lead to foam cell formation, irrespective of lipid peroxidation, including formation of complexes of aggregated $[41,42]$ or slightly modified LDL particles with antibodies against them [29]. Introduction of the latter complexes into macrophages, by way of the immunoglobulins (FC) receptor, results in foam cell formation [43]. Foam cell formation via these and other peroxidation-independent mechanisms is presently a topic of extensive investigations [44-46].

One of the most convincing lines of evidence for the involvement of oxidized LDL in cardio vascular disease is based on the clinically-validated association of various attributes of cardiovascular pathologies with the levels of oxidized phospholipids on apolipoprotein B (OxPL/apoB), as assayed in-vitro by the murine monoclonal antibody EO6 [47]. Using this assay, based on the specific binding of the EO6 antibody to the phosphocholine head group of oxidized phospholipids, it has been shown that the levels of oxidized phospholipids on circulating lipoproteins in the plasma:

- Are independent of traditional risk factors and the metabolic syndrome.

- Enhance the risk prediction of the Framingham Risk Score.

- Predict the presence and progression of coronary, femoral and carotid artery disease (particularly when amplified by lipoprotein(a) (Lp(a)) and phospholi- 
pases such as PLA2).

- Increased following acute coronary syndromes and percutaneous coronary intervention.

- Reflect the biological activity of Lp(a) particles.

- Provide diagnostic and prognostic information on the presence and progression of cardiovascular disease and clinically validated power to predict cardiovascular events.

In more than one respect, the immunological assay is a reliable tool to evaluate a well defined type of oxidative stress, as discussed below.

\section{APPARENT INCONSISTENCIES OF THE LDL OXIDATIVE MODIFICATION HYPOTHESIS OF AS WITH EXPERIMENTAL DATA}

\subsection{Are CVD Patients under OS?}

OS is an intuitively-defined term that describes a state of an excess of pro-oxidative factors over antioxidative factors [19]. In our analysis of available information on the $\mathrm{OS}$, as evaluated on the basis of various biomarkers, we found reasonable correlations between the OS, as measured by different methods when (and only when) the biomarkers measured similar processes [48]. Thus, the OS, as evaluated on the basis of different products of lipid peroxidation correlated with each other, and the same is true for different markers of DNA fragmentation but the estimates of OS based on factors of two different groups rarely correlate with each other. Based on this analysis, we concluded that oxidative stress cannot be defined by any universal index and proposed that these results indicate that there are different types of OS.

We do not believe that it is possible to define a universal criterion for oxidative stress, which can enable comparison of data from different laboratories using different methods. The term OS is ill-defined and covers a range of different types of context-dependent OS. Reasonable correlations have been observed between the OS determined on the basis of different tests of chemically similar factors. This justifies defining a criterion for each of these types but even this possibility is questionable because OS of any type may be local and either be reflected in available body fluids, or not.

Hence, comparison between the OS in one group of subjects (e.g. CVD patients) and another group (e.g. healthy people) is legitimate only in terms of the same biomarker. Figure 1 depicts the results of meta-analyses of the difference between the OS biomarkers in CVD patients and healthy people, as evaluated on the basis of different assays. The clear result is that the available data exhibit significant differences only when OS was estimated on the basis of the level of MDA [49]. Interestingly, the MDA concentrations in patients with stable

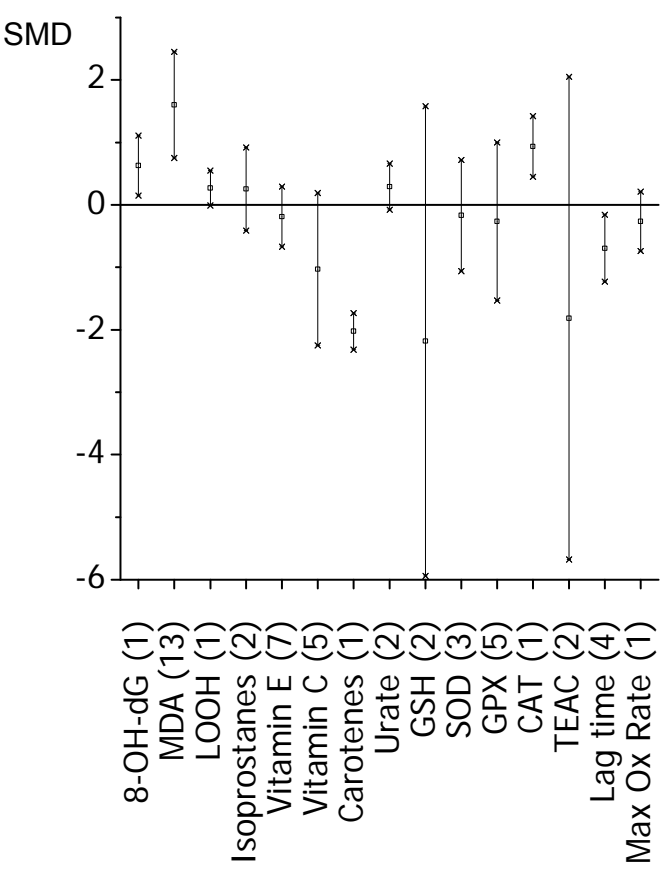

Figure 1. Indices of oxidative stress in cardiovascular disease (adopted from [49]). The pooled standardized mean difference (SMD) and the $95 \%$ confidence interval is given for each of the studied indices. Number of studies is given in parenthesis. Note that MDA is the only accepted index of oxidative stress that shows a mean difference greater than 1 SMD.

angina pectoris (SAP) are not different from matched controls, whereas unstable angina pectoris (UAP) patients have significantly higher MDA concentrations than both healthy controls and patients with SAP [49].

The observed differences between the different groups can not be taken as evidence that CVD patients are under oxidative stress, particularly because even the data based on MDA measurements is questionable due to: 1) the (questionable) reliability of the laboratory assay of MDA and 2) publication bias. This conclusion accords with that of Verhoye et al. [50], who noted that at present "published data linking oxLDL to cardiovascular disease cannot be compared because of the difference in the used assay protocol". Furthermore, the 'Asklepios Study' revealed that the results of oxLDL tests "show important variability and must be interpreted with caution". Taken together, the results demonstrate the complexity of the association between plasma oxLDL and CAD, to the extent that the oxLDL level was "independently associated with femoral plaque, but not carotid atherosclerosis".

In spite of these uncertainties, the trend is that oxidized phospholipids play a role in atherogenesis, as indicated by the immunologic assays described above. The role of oxidation in the pathogenesis of atherosclerosis, 
as commonly described may appears to be overestimated but this may reflect homeostatic mechanisms capable of reducing the effects of various factors on the oxidative stress, as described below.

\subsection{The Effect of Low Molecular Weight Antioxidants on Atherosclerosis}

Based on the free radical hypothesis of both longevity and atherosclerosis, supplementation of vitamin E could have been expected to have positive effects on both the rate of atherosclerosis and mortality. In fact, in animal models of atherosclerosis, antioxidants markedly decrease the rate of progression of lesions [28,51], in accordance with the oxidative modification hypothesis. By contrast, meta-analyses of the many published clinical trials show, at least apparently, higher mortality in vitamin E-treated people than in matched controls $[33,34]$. These meta-analyses induced serious critique (e.g. [5254]) and comprehensive responses to the critique $[55,56]$.

The criticism related mostly to the following issues:

1) The choice of clinical studies to be included in the meta-analyses.

2) The choice of mortality as the only end point.

3) The heterogeneity of the participants with respect to both population and treatment.

4) The model used to analyze the data (hierarchical logistic regression model vs. the traditional meta regression).

In short, in their communication in 2007, Blumberg and Frei described the "Clinical trials of vitamin E and cardiovascular diseases" as possibly being "fatally flawed" [54].

Decision Analysis [57,58] was designed to minimize the critique, by addressing these specific comments of the data as follows:

1) Inclusion of all the clinical trials quoted in the critique of the meta-analyses.

2) The use of quality-adjusted life years (QALY) [59] as an endpoint reflects the effects of supplementation on both mortality and morbidity.

3) Using Markov model-based Monte Carlo simulations enable adjustment for heterogeneities (both for population and treatment) on the basis of registries.

The main finding of this analysis was that vitamin $\mathrm{E}$ reduces the average QALY by almost four months but it also indicated that some people benefit from vitamin $\mathrm{E}$ supplementation [58]. Hence, the challenge was (and still is) to find criteria to differentiate between those who are likely to benefit from vitamin $\mathrm{E}$ supplementation and those who are not. Differentiation can be based either on identification of criteria that can be assayed in the lab or/and on data regarding the response of patients of specific diseases to supplementation (see below).

\subsection{The Effects of Vitamin E on HDL Peroxidation}

Oxidative modification of LDL is affected by HDL via different mechanisms, including acceleration of LDL peroxidation by HDL oxidation-products and inhibition of LDL peroxidation by removal of LDL peroxidation-products either by extraction from LDL to HDL and/or by their hydrolysis, catalyzed by HDL-associated enzymes. Hence, antioxidants can affect LDL peroxidation by altering the peroxidation of HDL and/or by altering the effect of HDL on LDL peroxidation. In the following discussion we first describe the results of a recent study that demonstrates the effect of vitamin E on HDL oxidation before relating to the mutual effects of peroxidation of HDL and LDL lipids.

The very complex effect of tocopherol on the susceptibility of lipoproteins to oxidation is demonstrated in the recent study of its effects on both in-vitro and ex-vivo peroxidation of HDL2 \& HDL3. Specifically, in their recent investigation, Wade et al. [60] found that the effect of added tocopherol depends on whether its peroxidation was tested before or after fractionation of the serum.

1) When added after fractionation, tocopherol protected the fraction of HDL2\&3 against peroxidation.

2) By contrast, when pre-incubated with non-fractionated serum and tested after fractionation, tocopherol promoted the peroxidation of HDL2\&3.

3) In another experiment, the authors tested the susceptibility of the HDL2\&3 fraction to peroxidation following 6-weeks of tocopherol supplementation. The main result of this experiment was that the HDL2\&3 fraction of the tocopherol-treated people were more susceptible to peroxidation than the same fraction of placebo-supplemented controls [60]. Interestingly, the increased sensitivity to peroxidation was accompanied by a decrease of the activity of both HDL2\&3-PON-1 and HDL2-LCAT (lecithin-cholesterol acyltransferase), probably because in the presence of higher concentrations of Toc, lower concentrations of the latter HDL-associated protective proteins were required, as described below.

To conclude, tocopherol can either protect or promote HDL oxidation, depending upon the timing of exposure of serum to tocopherol. In turn, HDL (and its oxidation products) affects LDL oxidation, thus altering the complex effects of tocopherol on atherogenesis, as described below.

\subsection{The Effect of HDL on LDL Peroxidation}

HDL, the beneficial form of blood cholesterol, (commonly denoted "the good cholesterol") is an established predictor of cardiovascular health. Its protective effect of the artery wall against atherosclerosis is well established. 
Furthermore, even in individuals whose LDL level is low, HDL remains a strong predictor of the risk of coronary artery disease (CAD), indicating that HDL is an independent risk factor [51,61]. The positive effects of HDL are commonly attributed to its ability to evacuate cholesterol from the periphery to the liver. In addition to this "reverse" transport, HDL plays a modulatory role in inflammation [62], which may also contribute to its cardioprotective effect. The importance of the antioxidative effect of HDL is emphasized by the findings of associations between impaired antioxidant activity of HDL and various diseases $[63,64]$.

In view of these facts, it is apparently surprising that under certain conditions, HDL oxidation accelerates LDL oxidation. Under such conditions, tocopherol-induced acceleration of HDL oxidation can be expected to promote LDL oxidation, thus promote atherogenesis. In view of the latter possibility, much effort has been devoted to investigate the mutual relationship between the oxidation of HDL and LDL, as described below.

\subsubsection{Lipid Peroxidation in Mixtures of LDL and HDL}

Under many conditions, HDL is more susceptible to oxidation than LDL $[65,66]$. This is not surprising in view of the similar or higher content of Vitamin E and other antioxidants, including beta-carotene, ubiquinol (coenzyme Q-10) and lycopene, in LDL. These antioxidants can act as the first line of defense. Furthermore, the higher surface /volume ratio in the smaller HDL particle can also accelerate the peroxidation induced by transition metal ions. It is also consistent with the finding that the smaller LDL particles are more readily oxidized than the larger ones [67]. However, the higher oxidizability of HDL is apparently inconsistent with the cardio-protective effect of HDL.

Systematic kinetic studies on the oxidation in mixtures of HDL and LDL demonstrated the complexity of the effect of HDL on LDL peroxidation (and vice versa). Specifically, these mutual effects [68] depend in a complex fashion on the composition and physical properties of both these lipoproteins as well as on the inducer of peroxidation and the concentrations of transition metal ions and several serum proteins.

The main results of the latter study were that:

1) Oxidation of LDL induced either by AAPH or by Myeloperoxidase (MPO) is inhibited by HDL under all the studied conditions, whereas.

2) Copper-induced peroxidation of LDL is inhibited by HDL at low copper/lipoprotein ratio but accelerated by HDL at high copper/lipoprotein ratio.

These results indicate that the antioxidative effects of HDL are only partially due to HDL-associated enzymes, in agreement with the finding that reconstituted HDL, containing no such enzymes, inhibits peroxidation induced by low copper concentration [68]. Reduction of the binding of copper to LDL by competitive binding to the HDL can also contribute to the antioxidative effect of HDL.

The acceleration of copper-induced oxidation of LDL by HDL, observed at high copper concentration, may be attributed to the hydroperoxides formed in the "more oxidizable" HDL, which can migrate to the "less oxidizable" LDL and enhance the oxidation of the LDL lipids induced by bound copper.

Given the sensitivity of the rate of oxidation to many factors, this hypothesis does not necessarily contradict previous results [69], observed under different conditions, in which the migration of hydroperoxides between lipoprotein particles was too slow to accelerate the peroxidation in "native lipoprotein particles". Notably, our interpretation of the acceleration of copper-induced LDL peroxidation by HDL is supported by the results of experiments in which native LDL was added to oxidizing lipoproteins at different time points. When the native LDL was added prior to decomposition of the hydroperoxides in the oxidizing lipoprotein, the lag preceding oxidation of the LDL was much shorter than the lag observed when the native LDL was added at later stages (Figure 2), after the level of hydroperoxides became reduced due to their copper-catalyzed decomposition [68].

Furthermore, this interpretation accords with the acceleration of LDL peroxidation upon sequential exposure to copper [70]. Altogether, the observed interrelationship between the oxidation of HDL and LDL and its dependence on the specific conditions should be considered in future investigations regarding the oxidation of lipoproteins.

\subsubsection{Mechanisms that Contribute to the Inhibition of LDL Peroxidation by HDL}

The oxidative damage caused by peroxidation products can be reduced by their evacuation from the site of their production, where they may accelerate the propagation of free radicals chain reaction. Accordingly, the mechanisms that can contribute to the net effect of HDL can be divided to two categorize:

1) Mechanisms based on transporting peroxidation products to the liver (here denoted "the physical mode").

2) Mechanisms based on metabolism, particularly hydrolysis of short or/and oxidized chain phospholipids, catalyzed by LDL-associated PAF acetyl hydrolase and HDL-associated enzymes, including PON-1, PON-2 (here denoted "the chemical mode").

In relating to the chemical mode, we recall that under certain conditions the LDL-associated PAF-acetyl-hydrolase from human plasma prevented oxidative modification of LDL [71], whereas in other experiments, 

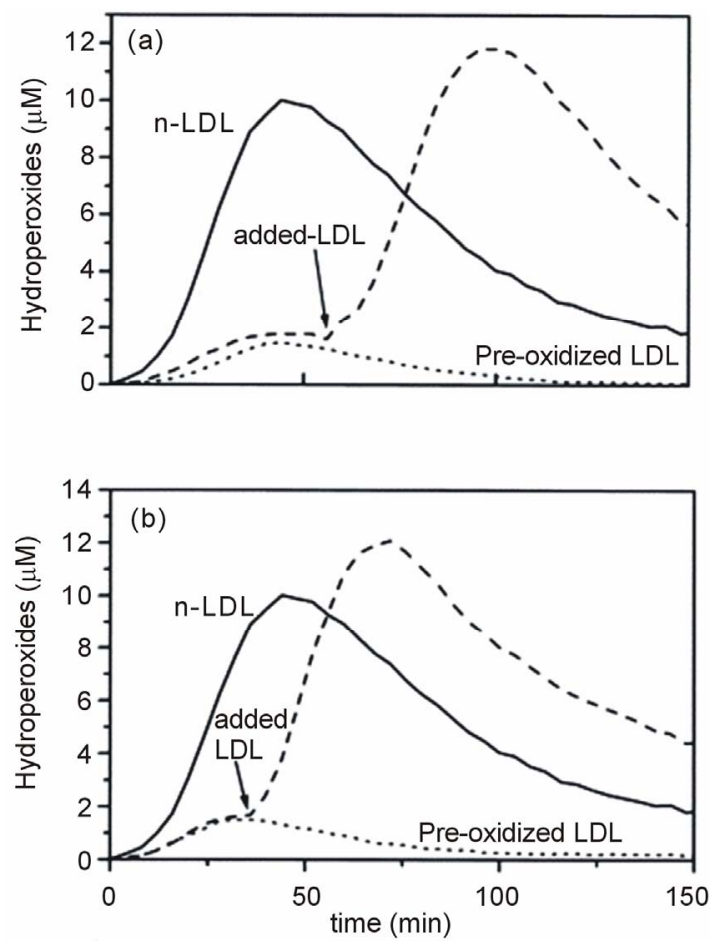

Figure 2. Sequential exposure of lipoproteins to copper-induced peroxidation (adopted from [68]). Accumulation of hydroperoxides during copperinduced oxidation at $37^{\circ} \mathrm{C}$ is described as a function of time. In both panels, the solid lines depict control peroxidation experiments, conducted with native LDL. The dashed lines in both panels depict sequential exposure experiments, in which LDL was added at the time points indicated by arrows, to a mixture of $\mathrm{CuCl}_{2}$ and either LDL (panel A) or HDL (panel B). The cholesterol concentration in the pre-exposed lipoproteins was a fifth of the concentration of the added LDL.

inhibition of this enzyme did not affect ex-vivo peroxidetion induced by either copper, Myeloperoxidase or AAPH [72]. All that this apparent contradiction means is that the effect of hydrolysis on the rate of peroxidation depends on the experimental conditions via a not yet known mechanism.

This is probably valid for other lipoprotein-associated enzymes. Our results accord with those of Teiber et al. [73], who showed that "Purified human serum PON1 does not protect LDL against oxidation in the in vitro assays initiated with copper or AAPH", in direct contrast to the results of Fyrnys et al. [74] and others. Teiber et al. attributed their results to the "removal of PON1 from its natural environment", which means that a method based on the antioxidative properties of highly purified PON1 may be irrelevant to the antioxidative properties of the HDL-associated enzymes. Although this interpretation may hold for the antioxidative activity of purified PON1, it does not explain our results regarding the effect of LDL-associated PAF-AH on the peroxidation in unfrac- tionated serum.

The role of PON has been extensively studied. In 1991 Mackness et al. [75] showed that PON is involved in the protection of LDL phospholipids against oxidation during the onset of the atherosclerosis process. Two years later, these authors demonstrated that PON is a very strong antioxidant, responsible for inhibition of HDL peroxidation and for the effect of HDL on LDL peroxidation [76]. These findings initiated extensive research of both the HDL-associated enzyme PON1 and the intracellular enzyme PON2, which is not carried via HDL. Both these enzymes are antioxidants and anti-atherogenic [77-81] probably due to prevention of the accumulation of lipid peroxides in HDL and LDL by PONcatalyzed metabolism of peroxidation products. Although there is no general consensus, the level of PON has been reported to be relatively low in many diseases, indicating that reduced levels of PON may contribute to these diseases. All we can conclude at this time is that we are far from understanding in detail the role of PONs.

In relating to the physical mode, it is important to recall that the level of peroxidizable lipids in LDL is equal or higher than in HDL. By contrast, the levels of both early and late oxidation products are higher in HDL than in LDL $[61,82]$. Thus, under conditions of continuing production of peroxyl radicals, HDL accumulated more lipid oxidation products than LDL. As mentioned above, one contributing factor is the higher levels of lipid-soluble antioxidants in LDL than in HDL. Notably, the effect of the lipoprotein-associated antioxidants is also affected by the concentration of water-soluble antioxidants due to replenishment of the lipid-soluble antioxidants. As an example, Vitamin $\mathrm{C}$ is soluble in water, but not in organic solvents or in lipids such as those found in LDL. Hence, it cannot act within the LDL particle. Nonetheless, vitamin $\mathrm{C}$ can reduce oxidized vitamin $\mathrm{E}$ at the water-lipid interface, so that the molecule of vitamin E can act once again as a protective agent. In this indirect way, vitamin C "cycles" the vitamin E within the LDL particle [83].

Another important factor is the relative rate of removal of peroxidation-products from the two lipoproteins via their enzymatic hydrolysis. This process is also dependent in a complex fashion on the rate of transfer of peroxidation products (and their hydrolysis products) between HDL and LDL. Under certain conditions, the transfer of lipid hydroperoxides appears to be too slow to substantially influence the distribution of these compounds in plasma [32], whereas under different conditions the initially formed (in HDL) hydroperoxides accelerate the peroxidation of LDL lipids [68]. The kinetic profile of lipid peroxidation in mixtures of HDL and LDL depends on the relative concentrations and susceptibilities of the two lipoproteins. Specifically, under cer- 
tain conditions it may be possible to observe HDL peroxidation followed by peroxidation of LDL lipids. Under different conditions, the different susceptibilities is not sufficiently large to observe two time dependencies and the time course of hydroperoxide accumulation appears to be relatively broad and the lag being either independent of the less oxidizable LDL or an increasing function of the LDL/HDL ratio [68].

\subsection{The Relative Susceptibility of Serum Lipoproteins to Peroxidation and Its Association with the Concentrations of Lipoprotein Fractions}

An intriguing result of our previous study is the highly significant positive correlation (p-value less than 0.001) between the HDL levels in the sera of hypercholesterolemic patients and the susceptibility of the serum lipids in unfractionated serum to copper-induced peroxidation ex-vivo, as evaluated on the basis of kinetic assay and expressed in terms of $t_{\max }$ [84] (Figure 3). We think that the lag preceding the rapid interrelated peroxidation of HDL and LDL reflects the ratio between the concentrations of the two lipoproteins. In other words, "HDL particles contain high amounts of lipid hydroperoxides [82] and thus, shorten the lag time and increase the amount of oxidized PUFAs", in agreement with the association of high HDL concentrations with LDL oxidation [66] and with the high correlation between serum oxidizability and HDL content found in the recent NMR study of Tynkkynen et al. [85].

In view of the slow development of atherogenesis and the possible protective role of HDL, we speculate that the latter contra-intuitive result can be attributed to a yet

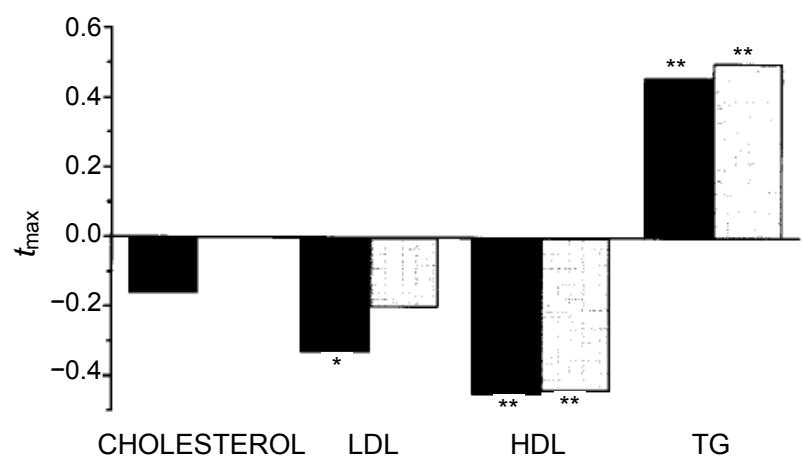

Figure 3. The relation between serum composition and oxidation parameters (adopted from [84]). Correlation coefficients are given between the serum concentrations of cholesterol (total), LDL cholesterol, HDL cholesterol, and total triglycerides (TG), on one hand, and $t_{\max }$, on the other. The black bars relate to oxidation kinetics as recorded at $245 \mathrm{~nm}$; the gray bars relate to the kinetics as recorded at $268 \mathrm{~nm}$. The symbol ${ }^{*}$ corresponds to a P-value smaller than 0.05 ; the symbol ${ }^{* *}$ corresponds to a $\mathrm{P}$-value smaller than 0.001 . unknown mechanism aimed at accelerating the removal of oxidative products via "reverse transport".

\section{WHAT IS RESPONSIBLE FOR THE APPARENT INCONSISTENCIES BETWEEN THE HYPOTHESIS AND THE EXPERIMENTAL RESULTS?}

Similar to many other investigations, the general approach to the "Oxidative modification hypothesis" was to test predictions based on the hypothesis. Specifically, the hypothesis attributing atherogenesis to oxidative modification led to the prediction that if the theory is correct, antioxidants should be cardio-protective. In cellular systems, the experimental results appeared to be consistent with this prediction. Furthermore, in cholesterol-fed animals, vitamin-E supplementation inhibited the atherogenesis, as predicted. Unfortunately, clinical trials yielded disappointing results, raising doubts regarding the causal relationships between $\mathrm{AS}$ and OS.

As in other cases of inconsistencies between the predicted and experimental results, several studies 'blamed the experiments'; others questioned the hypothesis. The question is often "which is wrong?" [86]. In fact, there is another possibility, which is that the hypothesis is right, the experiments are conducted correctly, but the predictions based on the hypothesis suffer from over-simplification. We think that the latter possibility is valid for the present issue. Specifically, the predictions were based on the "quench free radicals, prevent AS" approach, which can not be expected to be valid for this multifactorial process because it ignores many factors, including the possible pro-oxidative effects of "antioxidants", the possibility that the antioxidants may interfere with the synthesis of antioxidative enzymes, the possible reduction of the OS to levels lower than "normal", the contribution of oxidative modifications of LDL via non-radical mechanism(s) (e.g. [87]) and the possibility that ROS induces the process without being involved in later stages of atherogenesis, which means that vitamin E supplementation after the initiation of the process is too late [88].

In other words, we think that although lipid peroxidation is involved in atherogenesis, as indicated by many experiments, a causal relationship could not have been predicted on the basis of the limited available knowledge regarding the complex, multi-factorial, interdependent dependencies of atherogenesis on the "oxidative stress" and antioxidants.

\section{Antioxidants Do Not Always Inhibit LDL Peroxidation}

They may even accelerate it if/when:

1) The supplemented antioxidant acts as a pro-oxidant by reducing transition metal ions (e.g. vitamin C $[89,90]$ ) 
or via tocopherol-mediated peroxidation (TMP) [91].

2) The added antioxidant reduces the OS too much, to levels below normal, thus causing toxic effects (e.g. due to promotion of the adhesion of red blood cells to endothelial cells [92]).

3) Modified lipoproteins are formed via free radical-independent mechanisms $[46,87,93]$.

4) Free radicals initiate atherosclerosis but play only a minor role in later stages of the complex processes that yield AS, so that vitamin $\mathrm{E}$ does not alter the process when added after initiation occurred [88].

5) The antioxidant interferes with the protective effect of HDL against LDL peroxidation [60], as discussed above.

6) The antioxidant reduces the production of other antioxidative factors, as indicated by the slight difference between CVD patients and healthy controls. It is also consistent with the interpretation of Ristow et al. [7] to their disappointing results concerning the (negative) effects of vitamin E-supplementation on the health of sportsmen. Furthermore, this possibility is supported by the finding of high oxidizability of HDL in the serum of vitamin E-treated patients [60]. This may also be true for LDL and if it is not the case, we can still expect changes in LDL oxidation due to HDL oxidation, as described above.

Given this long (yet partial) list of possible reasons for the apparent contradictions between the observed and predicted relationship of oxidative modification and CVD, we can only conclude that this relationship is certainly uncertain.

\section{OS IN CVD, CONCLUDING REMARKS, IMPLICATIONS AND SPECULATIONS}

Atherogenesis is a "chronic" disease that develops during many years. It depends (at least partially) on alteration of the antioxidant-pro-oxidant balance, which is responsible for maintenance of the homeostatic level of the redox state. In comparison to the homeostatic level, OS means that the redox status in the relevant body compartment is "oxidative". Antioxidants may be sufficient to shift it to normal levels but too much antioxidant may cause a shift to a "reductive state". Alternative terminology can be based on the level of ROS in comparison to their homeostatic level. Specifically, OS may be described as being the result of "hyperOSemia"; an excess of antioxidants can be attributed to "hypoOSemia".

In fact, we have no data on the dependence of either the functions or the toxic effects of ROS on their concentrations. In other words, the dependence of AS on OS is not well understood. This does not undermine the validity of the hypothesis that peroxidation is responsible for atherogenesis [20] but it certainly justifies reconsideration of the hypothesis. To us, the present review indi- cates that ROS is not just the "bad guys" and not the only "bad guys" in the multi-factorial pathogenesis of coronary artery disease and atherogenesis.

The major change in the field of free radical research, particularly in biology and medicine, is the growing uncertainties. Now that we realize the importance of the tight control of homeostatic level of OS, the paradigm of "bad free radicals versus good antioxidants" can not be retained, not only with respect to cardiovascular issues. Even Harman's "free radical theory of aging" is questionable. This state of art (in Atherosclerosis) raises at least three questions: 1) what is the sequence of processes that lead to CVD and AS? 2) what is the interrelationship between LDL oxidation and CVD? and 3) who is likely to benefit from antioxidant supplementation?

While the first two questions have been investigated and partially answered $[44,45]$, we have no clues to answer the third [94]. Needless to say, high dose indiscriminate supplementation of vitamin E can not be recommended to the general public (that still spends millions of dollars on vitamin E supplements). Yet, it is quite clear that some specific groups may gain from vitamin $\mathrm{E}$ supplementation. The challenge is to define a criterion to predict who is likely to benefit from antioxidant supplementation.

In his commentary in 1989, Witztum raised the question "To E or not to E?" [95] and proposed that antioxidants can be expected to be beneficial for people under oxidative stress. He then pointed out that the problem is that we "lack measures to identify high-risk groups that would theoretically benefit most from antioxidant interventions" as well as a reliable measure "to determine the in vivo effectiveness of such interventions". Unfortunately, not only the existing data is still insufficient to predict who is likely to benefit from vitamin E treatment. We even don't know which type of oxidative stress has to be reduced. What we know is that based on the results of the population-based Cache County Study, Hayden et al. [52] advocate "further caution regarding the use of vitamin E by those with existing cardiovascular disease". This statement is inconsistent either with the assumption that people under oxidative stress can be expected to benefit from vitamin $\mathrm{E}$ or with the assumption that CVD patients are under high oxidative stress. We think that the apparent contradiction may be partially due to unrealistic estimation of the role of oxidative stress in CVD, in agreement with our conclusion regarding the (modest) role of OS in CVD patients [49].

Furthermore, in his 2003 communication, Frei raised the question of whether "to C or Not to C" and concluded that "What we know with certainty, however, is that a healthy diet and lifestyle lowers the risk of CHD, and this is what we should advocate to CHD patients and healthy people alike" [96]. Again, this kind of conclusions does not help in our goal of understanding the 
questions defined above. In short, the ill-defined term "oxidative stress" does not differentiate between the existence of "different types of oxidative stress" and the possibility that different antioxidants may have different antioxidative activity against different types of oxidative stress.

Another disappointing result is that physical exercise, which is likely to cause excessive oxidation (thus, OS), does not increase the benefit from antioxidant supplementation. In fact, antioxidants prevent health-promoting effects of physical exercise [7].

In our future search for criteria to predict who is likely to benefit from antioxidant supplementation, we can either look for people who are under high (relevant type of) OS or/and patients suffering from a disease that have been associated with OS such as dialysis patients [97] or diabetes mellitus type 2 (DM2) patients with haptaglobin 2-2 genotype [98].

As long as we do not have such criteria, we propose basing the decision of whether or not to expect benefit from chronic supplementation of antioxidants on whether or not a relatively short period (e.g. one month) of supplementation to a patient with low vitamin E level results in a significant decrease of the blood level of lipid peroxidation markers (e.g. MDA).

This approach is supported by a previous study of the effect of vitamin $\mathrm{E}$ on $\mathrm{AD}$ patients, in which the clinical outcome of a group of "vitamin E respondents", as determined after a short period of supplementation, was highly significant and excided by far the effect observed in the group of $\mathrm{AD}$ patients that were classified as "none-respondents". In their words [99] "the decision of whether or not to expect benefit from chronic vitamin $\mathrm{E}$ supplementation should be based on whether or not one month of vitamin E supplementation to patient with low vitamin $\mathrm{E}$ level results in a significant decrease of the blood level of lipid peroxidation markers".

Regardless of the possible effects of food supplements, fruits and vegetables are beneficial and those "many patients (that) view vitamins as a quick fix to compensate for poor eating habits [Tara Parker-Pope, The Wall Street Journal, March 20, 2006] should be advised of the difference between food ingredients and food supplements. A glass of red wine, orange juice or a salad of vegetables or fruits along with ROS-containing food is likely to prevent absorption of unhealthy peroxides by quenching ROS in the stomach [100]. Furthermore, it is tasty. Synthetic supplements, commonly do not "meet" toxic food ingredients in the gastrointestinal system. Maybe this is why they are not as beneficial as expected.

\section{ACKNOWLEDGEMENTS}

We thank Dr Edit Schnitzer and Prof Menachem Fainaru of TAU and Prof Yury Miller (UCSD) for their constructive remarks and the Lady
Davis Foundation for financial support.

\section{REFERENCES}

[1] Terman, A. and Brunk, U.T. (2006) Oxidative stress, accumulation of biological "garbage", and aging. Antioxidants \& Redox Signaling, 8, 197-204.

http://dx.doi.org/10.1089/ars.2006.8.197

[2] Droge, W. (2002) Free radicals in the physiological control of cell function. Physiological Reviews, 82, 47-95.

[3] Finkel, T. (1998) Oxygen radicals and signaling. Current Opinion in Cell Biology, 10, 248-253.

http://dx.doi.org/10.1016/S0955-0674(98)80147-6

[4] Halliwell, B. (2009) The wanderings of a free radical. Free Radical Biology and Medicine, 46, 531-542. http://dx.doi.org/10.1016/j.freeradbiomed.2008.11.008

[5] Halliwell, B., Gutteridge, J.M.C. and Cross, C.E. (1992) Free-radicals, antioxidants, and human-disease-Where are we now. Journal of Laboratory and Clinical Medicine, 119, 598-620.

[6] Rattan, S.I.S. and Demirovic, D. (2010) Hormesis can and does work in humans. Dose-Response, 8, 58-63. http://dx.doi.org/10.2203/dose-response.09-041.Rattan

[7] Ristow, M., Zarse, K., Oberbach, A., Kloting, N., Birringer, M., Kiehntopf, M., et al. (2009) Antioxidants prevent health-promoting effects of physical exercise in humans. Proceedings of the National Academy of Sciences of the United States of America, 106, 8665-8670. http://dx.doi.org/10.1073/pnas.0903485106

[8] Valko, M., Leibfritz, D., Moncol, J., Cronin, M.T.D., Mazur, M. and Telser, J. (2007) Free radicals and antioxidants in normal physiological functions and human disease. International Journal of Biochemistry \& Cell Biology, 39, 44-84.

http://dx.doi.org/10.1016/j.biocel.2006.07.001

[9] Halliwell, B. and Gutteridge, G.M.C. (2007) Free radicals in biology and medicine. Oxford University Press, Oxford.

[10] Martin, K.R. and Barrett, J.C. (2002) Reactive oxygen species as double-edged swords in cellular processes: Lowdose cell signaling versus high-dose toxicity. Human \& Experimental Toxicology, 21, 71-75. http://dx.doi.org/10.1191/0960327102ht213oa

[11] Yedgar, S., Krimsky, M., Cohen, Y. and Flower, R.J. (2007) Treatment of inflammatory diseases by selective eicosanoid inhibition: A double-edged sword? Trends in Pharmacological Sciences, 28, 459-464. http://dx.doi.org/10.1016/j.tips.2007.07.005

[12] Harman, D. (1956) Aging: A theory based on free radical and radiation chemistry. Journal of Gerontology, 11, 298300. http://dx.doi.org/10.1093/geronj/11.3.298

[13] Jang, Y.C. and Van Remmen, H. (2009) The mitochondrial theory of aging: Insight from transgenic and knockout mouse models. Experimental Gerontology, 44, 256260. http://dx.doi.org/10.1016/j.exger.2008.12.006

[14] Lyketsos, C.G., Steinberg, M., Tschanz, J.T., Norton, M.C., Steffens, D.C. and Breitner, J.C.S. (2000) Mental 
and behavioral disturbances in dementia: Findings from the Cache County Study on Memory in Aging. American Journal of Psychiatry, 157, 708-714. http://dx.doi.org/10.1176/appi.ajp.157.5.708

[15] Perez, V.I., Bokov, A., Van Remmen, H., Mele, J., Ran, Q.T., Ikeno, Y., et al. (2009) Is the oxidative stress theory of aging dead? Biochimica et Biophysica Acta-General Subjects, 1790, 1005-1014. http://dx.doi.org/10.1016/j.bbagen.2009.06.003

[16] Ristow, M. and Schmeisser, S. (2011) Extending life span by increasing oxidative stress. Free Radical Biology and Medicine, 51, 327-336. http://dx.doi.org/10.1016/j.freeradbiomed.2011.05.010

[17] Cheeseman, K.H. and Slater, T.F. (1993) An introduction to free-radical biochemistry. British Medical Bulletin, 49, 481-493.

[18] Gutteridge, J.M.C. (1993) Free-radicals in disease processes-A compilation of cause and consequence. Free Radical Research Communications, 19, 141-158. http://dx.doi.org/10.3109/10715769309111598

[19] Sies, H. (1991) Oxidative stress-From basic research to clinical-application. American Journal of Medicine, 91, S31-S38. http://dx.doi.org/10.1016/0002-9343(91)90281-2

[20] Steinberg, D., Parthasarathy, S., Carew, T.E., Khoo, J.C. and Witztum, J.L. (1989) Beyond cholesterol-Modifications of low-density lipoprotein that increase its atherogenicity. New England Journal of Medicine, 320, 915924.

[21] Brown, M.S. and Goldstein, J.L. (1986) A receptor-mediated pathway for cholesterol homeostasis. Science, 232, 34-47. http://dx.doi.org/10.1126/science.3513311

[22] Goldstein, J.L., Ho, Y.K., Basu, S.K. and Brown, M.S. (1979) Binding-site on macrophages that mediates uptake and degradation of acetylated low-density lipoprotein, producing massive cholesterol deposition. Proceedings of the National Academy of Sciences of the United States of America, 76, 333-337. http://dx.doi.org/10.1073/pnas.76.1.333

[23] Chisolm, G.M. and Steinberg, D. (2000) The oxidative modification hypothesis of atherogenesis: An overview. Free Radical Biology and Medicine, 28, 1815-1826. http://dx.doi.org/10.1016/S0891-5849(00)00344-0

[24] Quinn, M.T., Parthasarathy, S. and Steinberg, D. (1985) Endothelial cell-derived chemotactic activity for mouse peritoneal-macrophages and the effects of modified forms of low-density lipoprotein. Proceedings of the National Academy of Sciences of the United States of America, 82, 5949-5953. http://dx.doi.org/10.1073/pnas.82.17.5949

[25] Quinn, M.T., Parthasarathy, S., Fong, L.G. and Steinberg, D. (1987) Oxidatively modified low-density lipoproteins -A potential role in recruitment and retention of monocyte macrophages during atherogenesis. Proceedings of the National Academy of Sciences of the United States of America, 84, 2995-2998. http://dx.doi.org/10.1073/pnas.84.9.2995

[26] Steinberg, D., Berliner, J.A., Burton, G.W., Carew, T.E., Chait, A., Chisolm, G.M. et al. (1992) Antioxidants in the prevention of human atherosclerosis. Summary of the
Proceedings of a National Heart Lung and Blood Institute Workshop, 5-6 September 1991, Bethesda, Circulation, 85, 2338-2344. http://dx.doi.org/10.1161/01.CIR.85.6.2337

[27] Carew, T.E., Schwenke, D.C. and Steinberg, D. (1987) Antiatherogenic effect of probucol unrelated to its hypocholesterolemic effect-Evidence that antioxidants in vivo can selectively inhibit low-density-lipoprotein degradation in macrophage-rich fatty streaks and slow the progression of atherosclerosis in the Watanabe heritable hyperlipidemic rabbit. Proceedings of the National Academy of Sciences of the United States of America, 84, 7725-7729. http://dx.doi.org/10.1073/pnas.84.21.7725

[28] Verlangieri, A.J. and Bush, M.J. (1992) Effects of Dalpha-tocopherol supplementation on experimentally induced primate atherosclerosis. Journal of the American College of Nutrition, 11, 131-138.

[29] Witztum, J.L. and Steinberg, D. (2001) The oxidative modification hypothesis of atherosclerosis: Does it hold for humans? Trends in Cardiovascular Medicine, 11, 93102. http://dx.doi.org/10.1016/S1050-1738(01)00111-6

[30] Esterbauer, H., Rotheneder, M., Striegl, G., Waeg, G., Ashy, A., Sattler, W., et al. (1989) Vitamin-e and other lipophilic antioxidants protect Ldl against oxidation. Fett Wissenschaft Technologie-Fat Science Technology, 91, 316-324.

[31] Pryor, W.A. (2000) Vitamin E and heart disease: Basic science to clinical intervention trials. Free Radical Biology and Medicine, 28, 141-164.

http://dx.doi.org/10.1016/S0891-5849(99)00224-5

[32] Stocker, R. and Keaney, J.F. (2004) Role of oxidative modifications in atherosclerosis. Physiological Reviews, 84, 1381-1478. http://dx.doi.org/10.1152/physrev.00047.2003

[33] Bjelakovic, G., Nikolova, D., Simonetti, R.G. and Gluud, C. (2004) Antioxidant supplements for prevention of gastrointestinal cancers: A systematic review and meta-analysis. Lancet, 364, 1219-1228. http://dx.doi.org/10.1016/S0140-6736(04)17138-9

[34] Miller, E.R., Pastor-Barriuso, R., Dalal, D., Riemersma, R.A., Appel, L.J. and Guallar, E. (2005) Meta-analysis: High-dosage vitamin E supplementation may increase allcause mortality. Annals of Internal Medicine, 142, 37-46. http://dx.doi.org/10.7326/0003-4819-142-1-200501040-0 $\underline{0110}$

[35] Greenberg, E.R. (2005) Vitamin E supplements: Good in theory, but is the theory good? Annals of Internal Medicine, 142, 75-76.

http://dx.doi.org/10.7326/0003-4819-142-1-200501040-0 $\underline{0112}$

[36] Henriksen, T., Mahoney, E.M. and Steinberg, D. (1981) Enhanced macrophage degradation of low-density lipoprotein previously incubated with cultured endothelial cells: Recognition by receptors for acetylated low-density lipoproteins. Proceedings of the National Academy of Sciences of the United States of America, 78, 6499-6503. http://dx.doi.org/10.1073/pnas.78.10.6499

[37] Steinbrecher, U.P., Parthasarathy, S., Leake, D.S., Witztum, J.L. and Steinberg, D. (1984) Modification of low-density lipoprotein by endothelial-cells involves lipid-peroxidation 
and degradation of low-density lipoprotein phospholipids. Proceedings of the National Academy of Sciences of the United States of America, 81, 3883-3887. http://dx.doi.org/10.1073/pnas.81.12.3883

[38] Kita, T., Nagano, Y., Yokode, M., Ishii, K., Kume, N., Ooshima, A., Yoshida, H. and Kawai, C. (1987) Probucol prevents the progression of atherosclerosis in watanabe heritable hyperlipidemic rabbit, an animal-model for familial hypercholesterolemia. Proceedings of the National Academy of Sciences of the United States of America, 84, 5928-5931. http://dx.doi.org/10.1073/pnas.84.16.5928

[39] Vaya, J. (2013) The association between biomarkers in the blood and carotid plaque composition-focusing on oxidized lipids, oxysterols and plaque status. Biochemical Pharmacology, 86, 15-18. http://dx.doi.org/10.1016/j.bcp.2013.01.025

[40] Levitan, I., Volkov, S. and Subbaiah, P.V. (2010) Oxidized LDL: Diversity, patterns of recognition, and pathophysiology. Antioxidants \& Redox Signaling, 13, 39-75. http://dx.doi.org/10.1089/ars.2009.2733

[41] Frank, J.S. and Fogelman, A.M. (1989) Ultrastructure of the intima in WHHL and cholesterol-fed rabbit aortas prepared by ultra-rapid freezing and freeze-etching. Journal of Lipid Research, 30, 967-978.

[42] Khoo, J.C., Miller E., Mcloughlin P. and Steinberg D. (1988) Enhanced macrophage uptake of low-density lipoprotein after self-aggregation. Arteriosclerosis, 8, 348358. http://dx.doi.org/10.1161/01.ATV.8.4.348

[43] Lichtman, A.H., Binder C.J., Tsimikas S. and Witztum J.L. (2013) Adaptive immunity in atherogenesis: New insights and therapeutic approaches. Journal of Clinical Investigation, 123, 27-36. http://dx.doi.org/10.1172/JCI63108

[44] Halliwell, B. (2011) Free radicals and antioxidants-Quo vadis? Trends in Pharmacological Sciences, 32, 125-130. http://dx.doi.org/10.1016/j.tips.2010.12.002

[45] Curtiss, L.K. (2009) Reversing atherosclerosis? New England Journal of Medicine, 360, 1144-1146. http://dx.doi.org/10.1056/NEJMcibr0810383

[46] Lakota, K., Artenjak, A., Cucnik, S., Brguljan-Hitij, J., Cegovnik, B., Salobir, B. et al. (2012) Atherogenesis, inflammation and autoimmunity-An overview. In: Parthasarathy, S., Ed., Atherogenesis, InTech, 187-202. http://dx.doi.org/10.5772/26811

[47] Taleb, A., Witztum, J.L. and Tsimikas, S. (2011) Oxidized phospholipids on apoB-100-containing lipoproteins: A biomarker predicting cardiovascular disease and cardiovascular events. Biomarkers in Medicine, 5, 673-694. http://dx.doi.org/10.2217/bmm.11.60

[48] Dotan, Y., Lichtenberg, D. and Pinchuk, I. (2004) Lipid peroxidation cannot be used as a universal criterion of oxidative stress. Progress in Lipid Research, 43, 200-227. http://dx.doi.org/10.1016/j.plipres.2003.10.001

[49] Dotan, Y., Lichtenberg, D. and Pinchuk, I. (2012) Are CVD patients under oxidative stress? In: Parthasarathy, S., Ed., Atherogenesis, InTech, pp. 413-424. http://dx.doi.org/10.5772/28181

[50] Verhoye, E. and Langlois, M.R. (2009) Circulating oxi- dized low-density lipoprotein: A biomarker of atherosclerosis and cardiovascular risk? Clinical Chemistry and Laboratory Medicine, 47, 128-137. http://dx.doi.org/10.1515/CCLM.2009.037

[51] Jain, S.K., Mcvie, R., Meachum, Z.D. and Smith, T. (2000) Effect of LDL plus VLDL oxidizability and hyperglycemia on blood cholesterol, phospholipid and triglyceride levels in Type-I diabetic patients. Atherosclerosis, 149, 69-73. http://dx.doi.org/10.1016/S0021-9150(99)00308-1

[52] Hayden, K.M., Welsh-Bohmer, K.A., Wengreen, H.J., Zandi, P.P., Lyketsos, C.G. and Breitner, J.C.S. (2007) Risk of mortality with vitamin E supplements: The cache county study. American Journal of Medicine, 120, 180184. http://dx.doi.org/10.1016/j.amjmed.2006.03.039

[53] Jialal, I. and Devaraj, S. (2005) High-dosage vitamin E supplementation and all-cause mortality. Annals of Internal Medicine, 143, 155.

http://dx.doi.org/10.7326/0003-4819-143-2-200507190-0 $\underline{0026}$

[54] Blumberg, J.B. and Frei, B. (2007) Why clinical trials of vitamin $\mathrm{E}$ and cardiovascular diseases may be fatally flawed. Commentary on "The relationship between dose of vitamin E and suppression of oxidative stress in humans". Free Radical Biology and Medicine, 43, 1374-1376. http://dx.doi.org/10.1016/j.freeradbiomed.2007.08.017

[55] Bjelakovic, G., Nikolova, D., Gluud, L.L., Simonetti, R.G. and Gluud, C. (2007) Mortality in randomized trials of antioxidant supplements for primary and secondary prevention: Systematic review and meta-analysis. JAMA-Journal of the American Medical Association, 297, 842-857. http://dx.doi.org/10.1001/jama.297.8.842

[56] Miller, E.R., Appel, L.J., Guallar, E. and Pastor-Barriuso, R. (2005) High-dosage vitamin E supplementation and allcause mortality-Response. Annals of Internal Medicine, 143, 156-158.

http://dx.doi.org/10.7326/0003-4819-143-2-200507190-0 $\underline{0029}$

[57] Dotan, Y., Lichtenberg, D. and Pinchuk, I. (2009) No evidence supports vitamin E indiscriminate supplementation. Biofactors, 35, 469-473. http://dx.doi.org/10.1002/biof.61

[58] Dotan, Y., Pinchuk, I., Lichtenberg, D. and Leshno, M. (2009) Decision analysis supports the paradigm that indiscriminate supplementation of vitamin E does more harm than good. Arteriosclerosis Thrombosis and Vascular Biology, 29, 1304-1309.

http://dx.doi.org/10.1161/ATVBAHA.108.178699

[59] Muennig, P.A. and Gold, M.R. (2001) Using the yearsof-healthy-life measure to calculate QALYs. American Journal of Preventive Medicine, 20, 35-39. http://dx.doi.org/10.1016/S0749-3797(00)00261-0

[60] Wade, L., Nadeem, N., Young, I.S., Woodside, J.V., McGinty, A., McMaster, C. and McEneny, J. (2013) $\alpha$-tocopherol induces proatherogenic changes to $\mathrm{HDL}_{2} \& \mathrm{HDL}_{3}$ : An in vitro and ex vivo investigation. Atherosclerosis, 226, 392-397. http://dx.doi.org/10.1016/j.atherosclerosis.2012.11.032

[61] Shao, B. and Heinecke, J.W. (2009) HDL, lipid peroxidetion, and atherosclerosis. Journal of Lipid Research, 50, 599-601. http://dx.doi.org/10.1194/j1r.E900001-JLR200 
[62] Navab, M., Reddy, S.T., Van Lenten, B.J. and Fogelman A.M. (2011) HDL and cardiovascular disease: Atherogenic and atheroprotective mechanisms. Nature Reviews Cardiology, 8, 222-232.

http://dx.doi.org/10.1038/nrcardio.2010.222

[63] Toker, A., Kadi, M., Yildirim, A.K., Aksoy, H. and Akcay, F. (2009) Serum lipid profile paraoxonase and arylesterase activities in psoriasis. Cell Biochemistry and Function, 27, 176-180. http://dx.doi.org/10.1002/cbf.1553

[64] Moradi, H., Pahl, M.V., Elahimehr, R. and Vaziri, N.D. (2009) Impaired antioxidant activity of high-density lipoprotein in chronic kidney disease. Translational Research, 153, 77-85. http://dx.doi.org/10.1016/j.trsl.2008.11.007

[65] Raveh, O., Pinchuk, I., Schnitzer, E., Fainaru, M., Schaffer, Z. and Lichtenberg, D. (2000) Kinetic analysis of copper-induced peroxidation of HDL, autoaccelerated and tocopherol-mediated peroxidation. Free Radical Biology and Medicine, 29, 131-146. http://dx.doi.org/10.1016/S0891-5849(00)00332-4

[66] Brizzi, P., Tonolo, G., Carusillo, F., Malaguarnera, M., Maioli, M. and Musumeci, S. (2003) Plasma lipid composition and LDL oxidation. Clinical Chemistry and Laboratory Medicine, 41, 56-60. http://dx.doi.org/10.1515/CCLM.2003.010

[67] Chait, A., Brazg, R.L., Tribble, D.L. and Krauss, R.M. (1993) Susceptibility of small, dense, low-density lipoproteins to oxidative modification in subjects with the atherogenic lipoprotein phenotype, pattern-B. American Journal of Medicine, 94, 350-356.

http://dx.doi.org/10.1016/0002-9343(93)90144-E

[68] Raveh, O., Pinchuk, I., Fainaru, M. and Lichtenberg, D. (2001) Kinetics of lipid peroxidation in mixtures of HDL and LDL, mutual effects. Free Radical Biology and Medicine, 31, 1486-1497. http://dx.doi.org/10.1016/S0891-5849(01)00730-4

[69] Ishigaki, Y., Katagiri, H., Gao, J., Yamada, T., Imai, J., Uno, K., et al. (2008) Impact of plasma oxidized low-density lipoprotein removal on atherosclerosis. Circulation, 118, $75-83$.

http://dx.doi.org/10.1161/CIRCULATIONAHA.107.7451 $\underline{74}$

[70] Schnitzer, E., Fainaru, M. and Lichtenberg, D. (1995) Oxidation of low-density-lipoprotein upon sequential exposure to copper ions. Free Radical Research, 23, 137149. http://dx.doi.org/10.3109/10715769509064028

[71] Stafforini, D.M., Zimmerman, G.A., Mcintyre, T.M. and Prescott, S.M. (1992) The Paf acetylhydrolase from human plasma prevents oxidative modification of low-density-lipoprotein. Clinical Research, 40, A253.

[72] Schnitzer, E., Pinchuk, I., Fainaru, M., Lichtenberg, D. and Yedgar, S. (1998) LDL-associated phospholipase A does not protect LDL against lipid peroxidation in vitro. Free Radical Biology and Medicine, 24, 1294-1303. http://dx.doi.org/10.1016/S0891-5849(97)00454-1

[73] Teiber, J.F., Draganov, D.I. and La Du, B.N. (2004) Purified human serum PON1 does not protect LDL against oxidation in the in vitro assays initiated with copper or AAPH. Journal of Lipid Research, 45, 2260-2268. http://dx.doi.org/10.1194/jlr.M400213-JLR200
[74] Fyrnys, B., Blencowe, C. and Deigner, H.P. (1995) Susceptibility of phospholipids of oxidizing LDL to enzymatic-hydrolysis modulates uptake by $\mathrm{P}_{38} 8 \mathrm{D}_{1}$ macrophagelike cells. FEBS Letters, 357, 7-12. http://dx.doi.org/10.1016/0014-5793(94)01312-O

[75] Mackness, M.I., Arrol, S. and Durrington, P.N. (1991) Paraoxonase prevents accumulation of lipoperoxides in lowdensity-lipoprotein. FEBS Letters, 286, 152-154. http://dx.doi.org/10.1016/0014-5793(91)80962-3

[76] Mackness, M.I., Abbott, C., Arrol, S. and Durrington, P.N. (1993) The Role of high-density-lipoprotein and lipid-soluble antioxidant vitamins in inhibiting low-density lipoprotein oxidation. Biochemical Journal, 294, 829-834.

[77] Aviram, M., Hardak, E., Vaya, J., Mahmood, S., Milo, S., Hoffman, A., et al. (2000) Human serum paraoxonases (PON1) Q and R selectively decrease lipid peroxides in human coronary and carotid atherosclerotic lesions PON1 esterase and peroxidase-like activities. Circulation, 101, 2510-2517. http://dx.doi.org/10.1161/01.CIR.101.21.2510

[78] Mackness, M.I., Mackness, B., Durrington, P.N., Fogelman, A.M., Berliner, J., Lusis, A.J., et al. (1998) Paraoxonase and coronary heart disease. Current Opinion in Lipidology, 9, 319-324. http://dx.doi.org/10.1097/00041433-199808000-00006

[79] Mackness M.I., Durrington P.N. and Mackness B. (2000) How high-density lipoprotein protects against the effects of lipid peroxidation. Current Opinion in Lipidology, 11, 383-388. http://dx.doi.org/10.1097/00041433-200008000-00007

[80] Rosenblat, M. and Aviram, M. (2009) Paraoxonases role in the prevention of cardiovascular diseases. Biofactors, 35, 98-104. http://dx.doi.org/10.1002/biof.16

[81] Ng, C.J., Wadleigh, D.J., Gangopadhyay, A., Hama, S., Grijalva, V.R., Navab, M., Fogelman, A.M. and Reddy, S.T. (2001) Paraoxonase-2 is a ubiquitously expressed protein with antioxidant properties and is capable of preventing cell-mediated oxidative modification of low density lipoprotein. Journal of Biological Chemistry, 276, 44444-44449. http://dx.doi.org/10.1074/jbc.M105660200

[82] Bowry, V.W., Stanley, K.K. and Stocker, R. (1992) Highdensity-lipoprotein is the major carrier of lipid hydroperoxides in human blood-plasma from fasting donors. Proceedings of the National Academy of Sciences of the United States of America, 89, 10316-10320. http://dx.doi.org/10.1073/pnas.89.21.10316

[83] Buettner, G.R. (1993) The pecking order of free-radicals and antioxidants: Lipid-peroxidation, $\alpha$-tocopherol, and ascorbate. Archives of Biochemistry and Biophysics, 300, 535-543. http://dx.doi.org/10.1006/abbi.1993.1074

[84] Shimonov, M., Pinchuk, I., Bor, A., Beigel, I., Fainaru, M., Rubin, M., et al. (1999) Susceptibility of serum lipids to copper-induced peroxidation correlates with the level of high density lipoprotein cholesterol. Lipids, 34, 255-259. http://dx.doi.org/10.1007/s11745-999-0361-0

[85] Tynkkynen, T., Mursu, J., Nurmi, T., Tuppurainen, K., Laatikainen, R. and Soininen, P. (2012) NMR protocol for determination of oxidation susceptibility of serum lipids and application of the protocol to a chocolate study. Metabolomics, 8, 386-398. 
http://dx.doi.org/10.1007/s11306-011-0323-2

[86] Williams, K.J. and Fisher, E.A. (2005) Oxidation, lipoproteins, and atherosclerosis: Which is wrong, the antioxidants or the theory? Current Opinion in Clinical Nutrition and Metabolic Care, 8, 139-146. http://dx.doi.org/10.1097/00075197-200503000-00006

[87] Wu, B.J., Kathir, K., Witting, P.K., Beck, K., Choy, K., Li, C., et al. (2006) Antioxidants protect from atherosclerosis by a heme oxygenase-1 pathway that is independent of free radical scavenging. Journal of Experimental Medicine, 203, 1117-1127. http://dx.doi.org/10.1084/jem.20052321

[88] Niki, E. (2011) Do free radicals play causal role in atherosclerosis? Low density lipoprotein oxidation and vitamin E revisited. Journal of Clinical Biochemistry and Nutrition, 48, 3-7. http://dx.doi.org/10.3164/jcbn.11-007FR

[89] Podmore, I.D., Griffiths, H.R., Herbert, K.E., Mistry, N., Mistry, P. and Lunec, J. (1998) Vitamin C exhibits prooxidant properties. Nature, 392, 559. http://dx.doi.org/10.1038/33308

[90] Nyyssonen, K., Porkkala Sarataho, E., Kaikkonen, J. and Salonen, J.T. (1997) Ascorbate and urate are the strongest determinants of plasma antioxidative capacity and serum lipid resistance to oxidation in Finnish men. Atherosclerosis, 130, 223-233. http://dx.doi.org/10.1016/S0021-9150(96)06064-9

[91] Bowry, V.W. and Stocker, R. (1993) Tocopherol-mediated peroxidation. The prooxidant effect of vitamin $\mathrm{E}$ on the radical-initiated oxidation of human low-density-lipoprotein. Journal of the American Chemical Society, 115, 6029-6044. http://dx.doi.org/10.1021/ja00067a019

[92] Koshkaryev, A., Barshtein, G. and Yedgar, S. (2010) Vitamin E. induces phosphatidylserine externalization and red cell adhesion to endothelial cells. Cell Biochemistry and Biophysics, 56, 109-114.

http://dx.doi.org/10.1007/s12013-009-9074-3

\section{ABBREVIATIONS AND TERMS}

AAPH-2,2'-Azobis(2-amidinopropane) dihydrochloride; a water-soluble generator of free radicals;

AS-atherosclerosis;

MDA — malondialdehyde; a relatively stable peroxidation product;

PAF-AH - platelet aggregation factor acetyl hydrolase; PON-paraoxonase;

HDL associated PLA - the enzyme that catalyzes the hydrolysis of peroxidation products;

QALY — quality adjusted life years;

OS - oxidative stress; an ill-defined term commonly used to describe intuitively the imbalance between prooxidative and antioxidative species;

$\mathrm{RO}(\mathrm{N}) \mathrm{S}$ - reactive oxygen (and nitrogen) species; used when we do not know which reactive oxygen (or nitrogen) species are involved;
[93] Manea, A. (2012) Vascular biology of reactive oxygen species and NADPH oxidases: Role in atherogenesis. In: Parthasarathy, S., Ed., Atherogenesis, InTech, 425-446. http://dx.doi.org/10.5772/25368

[94] Lichtenberg, D. (2011) Who is likely to gain from high dose supplementation of vitamin E? Harefuah (heb), 150, $37-40$.

[95] Witztum, J.L. (1998) To E or not to E-How do we tell? Circulation, 98, 2785-2787. http://dx.doi.org/10.1161/01.CIR.98.25.2785

[96] Frei, B. (2003) To C or not to C, that is the question! Journal of the American College of Cardiology, 42, 253-255. http://dx.doi.org/10.1016/S0735-1097(03)00574-6

[97] Boaz, M., Smetana, S., Weinstein, T., Matas, Z., Gafter, U., Iaina, A., et al. (2000) Secondary prevention with antioxidants of cardiovascular disease in endstage renal disease (SPACE): Randomised placebo-controlled trial. Lancet, 356, 1213-1218. http://dx.doi.org/10.1016/S0140-6736(00)02783-5

[98] Blum, S., Vardi, M., Brown, J.B., Russell, A., Milman, U., Shapira, C., Levy, N.S., Miller-Lotan, R., Asleh, R. and Levy, A.P. (2010) Vitamin E reduces cardiovascular disease in individuals with diabetes mellitus and the haptoglobin 2-2 genotype. Pharmacogenomics, 11, 675-684. http://dx.doi.org/10.2217/pgs.10.17

[99] Lloret, A., Badia, M.C., Mora, N.J., Pallardo, F.V., Alonso, M.D. and Vina, J. (2009) Vitamin E paradox in Alzheimer's disease: It does not prevent loss of cognition and may even be detrimental. Journal of Alzheimer's Disease, 17, 143-149.

[100] Kanner, J., Gorelik, S., Roman, S. and Kohen, R. (2012) Protection by polyphenols of postprandial human plasma and low-density lipoprotein modification: The stomach as a bioreactor. Journal of Agricultural and Food Chemistry, 60, 8790-8796. http://dx.doi.org/10.1021/jf300193g

FR - free radicals; used when we relate to established free radical mechanism(s);

oxLDL-oxidatively-modified LDL; used when we mean that the relevant active species is one or more LDL oxidation product(s).

\section{HIGHLIGHTS}

- OS is tightly controlled. In CVD patients, it is only slightly higher than normal.

- The weak association of OS with CVD is insufficient to prove causal relationship.

- Oxidative modification of LDL is one of several processes that govern atherosclerosis.

- Antioxidants do not necessarily inhibit the multifactorial processes resulting in AS.

- Our knowledge is insufficient to predict who is likely to gain from antioxidants. 